\title{
Calculating Sensory Dissonance: Some Discrepancies Arising from the Models of Kameoka \& Kuriyagawa, and Hutchinson \& Knopoff
}

\author{
Keith Mashinter \\ UNIVERSITY OF WATERLOO ${ }^{[1]}$
}

\begin{abstract}
The phenomena of consonance and dissonance are thought to involve both learned and innate components. Work by Greenwood (1961) and Plomp and Levelt (1965) established that an aspect of dissonance perception can be traced to unique physiological properties of the hearing organ. This aspect of dissonance is commonly referred to as sensory dissonance. Two computable models of sensory dissonance are described and discussed — those of Kameoka and Kuriyagawa (1969a; 1969b) and Hutchinson and Knopoff (1978). Software implementations of both models are provided, and their behaviors explored. Both models exhibit a number of conceptual and technical problems.
\end{abstract}

Submitted 2006 February 7; accepted 2006 February 19

KEYWORDS: consonance, dissonance, sensory, modelling, perception

\section{INTRODUCTION}

WHAT accounts for the sense of "euphoniousness" or "harshness" of sounds? The concepts of consonance and dissonance have produced a long and complex history of music theorizing (see Tenney, 1988). Where early writers tended to link consonance and dissonance to acoustical or numerological notions, writers in the twentieth century were more likely to link consonance and dissonance with social and cultural views (see, for example, Cazden, 1980). Among current theorists, the phenomena of consonance and dissonance are thought to involve both culturally learned as well as innate components.

Pythagorus is credited with the discovery that tones whose frequencies are related by simple ratios are more consonant. In 1638 Galileo Galilei postulated that these simple ratios give rise to regular motions of the eardrum: less pleasant sensations arise when the eardrum moves in a more irregular fashion. Hermann von Helmholtz (1863) was perhaps the first researcher to take into account the fact that musical tones consist of many spectral components and that dissonance must also be influenced by timbre or tone color. Helmholtz argued that consonance comes not just from simple frequency ratio relationships, but also from the close coincidence of upper partials in typical sounds. In 1898, Carl Stumpf proposed that consonance arises from tonal fusion - the tendency for two tones to sound as one; however, this notion is not consistent with perceptual evidence suggeting that increasing the perceived number of sound sources produces a decrease in judged dissonance. Albert Bregman (1990) has drawn attention to the past confusion between sensory consonance ("smooth" sounding) and tonal fusion ("sounding as one"). A notable affirmation of this distinction is found in Huron (1991) who carried out a statistical analysis of a sample of music by J. S. Bach. Bach avoids tonal fusion while pursuing tonal consonance; in practical terms, he prefers thirds and sixths over octaves, fourths, and fifths.

In the latter half of the twentieth century, more precise psychoacoustic experiments and computer modelling enabled researchers to take a much closer look at dissonance. Greenwood's seminal research (1961a; 1961b) supported Helmholtz's theory but linked it to the critical band. Greenwood's work was extended by Plomp and Levelt (1965) who produced dissonance ratings based on critical bands that agreed with Malmberg's collected opinions (1918). These rated the octave (1:2), fifth (2:3), and fourth (3:4) as most consonant and on down to a minor second $(15: 16)$ as the most dissonant. Kameoka \& Kuriyagawa (1969a; 
1969b) and Hutchinson \& Knopoff (1978; 1979) developed dissonance estimating algorithms based on psychoacoustic experiments with human subjects and extrapolations of theory. In this article, implementations of both algorithms are described. In making sense of these algorithms, however, we will see that several problems arise. In light of these problems, some new directions for future research will be proposed.

Consonance is to cold as dissonance is to heat-one can imagine a state of zero heat and note its increase, but zero coldness is ill-defined since one could conjecture an infinite amount of heat. The term consonance is often more useful than lack of dissonance, just as cool is a more useful term than lack of heat. Consonance is usually identified with being pleasant, clear, or euphonious, while dissonance is regarded as harsh or turbid.

The psychoacoustic concept of the critical band plays an important role in modern conceptions of how we perceive dissonance. Von Békésy $(1939,1949)$ showed that different frequencies produce different points of maximum displacement on the basilar membrane. Low frequencies cause the greatest displacement toward the thicker furthest end of the membrane, its apex. Zwicker, Flottorp, \& Stevens (1957) developed a curve to represent critical bandwidth as a function of frequency, but it did not well represent low-frequency data. Critical bands are regions of excitation on the basilar membrane of the cochlea about $1 \mathrm{~mm}$ in size. Two frequencies that give rise to maximum excitation on the basilar membrane within the same critical band will cause mutual interference. Donald Greenwood (1961a; 1961b) was the first to draw attention to the relationship between sensory consonance/dissonance and critical bands. Of the many proposed curves for critical bandwidth, Greenwood's original function $F=A\left(10^{a x}-k\right)$ remains one of the best representations, where $F$ is the frequency in $\mathrm{Hz}, x$ is the position of maximum displacement in $\mathrm{mm}$ from the apex, and $A, a$, and $k$ are constants $(165,0.06$, and 1.0 respectively for humans). This curve, however, does not account for the effect of sound pressure level on critical bandwidth (Kameoka \& Kuriyagawa, 1969a).

\section{KAMEOKA AND KURIYAgAWA}

Akio Kameoka and Mamora Kuriyagawa used empirical data gathered from test subjects to formulate a computable model of relative and absolute dissonance. Both musicians and non-musicians were used as subjects in experiments to determine the effects of frequency and sound pressure level (SPL) on the perception of sensory dissonance in dyads of simple sine tones (Kameoka \& Kuriyagawa, 1969a). In a subsequent study (Kameoka \& Kuriyagawa, 1969b) they extended their theory to complex tones of various spectral components. Although they acknowledge Plomp and Levelt (Plomp \& Levelt, 1965) and Zwicker (1974, 1957) for their work with critical bands, Kameoka and Kuriyagawa base their model almost entirely on their own empirical data so that the results are largely independent of previous conceptualizations.

\subsection{Part I: Dissonance of Dyads}

Kameoka and Kuriyagawa's listening experiments result in a V-shaped curve of increasing/decreasing dissonance when plotted against a logarithmic ratio of frequency deviation (see Figure 1). The curve shown in Figure 1 traces changes of consonance/dissonance as the frequency separation moves from a unison to an octave. Kameoka and Kuriyagawa refer to the initial downward slope of the V as the "dynamic domain" and the upward-sloping part of the V as the "static domain". The downward slope is "dynamic" since this is the region where beating is most audible. The bottom of the $\mathrm{V}$ is the point of least consonance (greatest dissonance), corresponding to a frequency separation of about $10 \%(484 \mathrm{~Hz}$ ) if the lower tone is A $440 \mathrm{~Hz}$ at $57 \mathrm{~dB}$ SPL.

Although their initial experiments showed that subjects acknowledged some dissonance when presented with pure tones beyond an octave separation (Kameoka \& Kuriyagawa, 1969a, Fig. 5), Kameoka and Kuriyagawa neglect this supra-octave dissonance in their remaining experiments. Beyond the distance of one octave, Kameoka and Kuriyagawa assume a constant nominal dissonance. For a simple tone at $\mathrm{A}=440 \mathrm{~Hz}$, the most dissonant frequency according to Kameoka and Kuriyagawa model is $484 \mathrm{~Hz}$ if both tones are at $57 \mathrm{~dB}$ SPL. Their discounting of possible supra-octave dissonance was probably motivated by the practical desire to avoid the additional computations this would incur. Future implementations of their model could make use of this additional information, but the implementation presented here does not. 
Kameoka and Kuriyagawa note that the percent difference between the base frequency and the frequency of greatest dissonance decreases as the base frequency increases (Kameoka \& Kuriyagawa, 1969a, Fig. 6), which is consistent with Greenwood's observation of the link between dissonance and critical bands.

Kameoka and Kuriyagawa scale dissonance in two ways: relative dissonance and absolute dissonance. Relative dissonance is defined using $440 \mathrm{~Hz}$ and $484 \mathrm{~Hz}$ at $57 \mathrm{~dB}$ SPL as the most dissonant dyad with a dissonance of 100 units, and unison pure tones at $440 \mathrm{~Hz}, 60 \mathrm{~dB}$ SPL as the zero dissonance. Absolute dissonance (AD) is a constant multiple of the relative dissonance (RD) plus a constant related to ambient noise:

$$
\mathrm{AD}=k_{0} \mathrm{RD}+C_{0}
$$

The absolute dissonance scale is defined by setting $k_{0}=1.0$ and $C_{0}=65$. Thus the relative dissonance scale [0-100] maps to [65-165] in the absolute dissonance scale. Zero absolute dissonance is only reached when there are no external or internal noises and the total sound pressure level is zero.

The model first considers the frequency separation of the dyad and the SPL of the lower frequency to find the absolute dissonance. The higher partial's SPL is then used to adjust the absolute dissonance.

Kameoka and Kuriyagawa's experiments show that SPL does have an appreciable effect on the perception of dissonance, which is contrary to the popular theory of Zwicker, Flottorp, and Stevens (1957). Later studies by Greenwood (1961a; 1961b) and Glasberg \& Moore (1990) still only consider critical bandwidth as a function of frequency, but Vos (1986) acknowledges the importance of SPL in determining the width of critical bands.

\subsection{Part II: Calculation of Dissonance for Complex Tones}

The second part of Kameoka and Kuriyagawa's study deals with complex tones instead of pure sine tones. They propose that dissonance is additive and dependent on loudness, using the power law of psychological significance to combine the dissonance intensity dyads of harmonics of complex tones, resulting in a measure of "absolute dissonance". The power law described in Zwicker, Flottorp, and Stevens (1957) proposes that psychological magnitude $\psi$ can be expressed in terms of physical magnitude $\phi$ :

$$
\psi=k \phi^{\beta}
$$

where $k$ is a constant depending on the scale unit of $\psi$, and $\beta$ is a constant consistent with the sensation (Kameoka \& Kuriyagawa, 1969b, p. 1461). The loudness sensation, for example, uses $\beta$ as approximately .30 or .27. Kameoka and Kuriyagawa coin the term "dissonance intensity" to refer to psychological dissonance, so that the absolute dissonance of a dyad $D_{2 i}$ is related to its psychological dissonance intensity as follows:

$$
D_{2 i}=k_{0} D_{I 2 i}^{\beta}
$$

The additivity is done in the realm of intensities, which can be seen at the end of this interpretation of the model.

\subsection{Interpretation and Amendments}

In the following formulae, the lower tone of the dyad has frequency $f_{1}$ in $\mathrm{Hz}$, loudness $L_{1}$ in $\mathrm{dB}$ SPL, and pressure $p_{1}$ in $\mu$ bar. ${ }^{[2]}$ The difference between the base frequency $f_{1}$ and the most dissonant frequency above $f_{1}$ is given by (4) where $L_{1}$ is the sound pressure level in $\mathrm{dB}$. Note that (4) does not apply for SPLs under 17 $\mathrm{dB}$ SPL, a nominal level for human aural perception.

$$
f_{b}=2.27\left(\frac{L_{1}-57}{40}+1\right) f_{1}^{0.477}
$$

The absolute dissonance of dyads is given by the following equations, which are derived from the idealized V-curves from Kameoka \& Kuriyagawa (1969a). Kameoka and Kuriyagawa failed to document the case when $\left(f_{2}-f_{1}\right) / f_{1} \leq 0.01$. In this range, equation (6) is not suitable since $2+\log _{10} 0.01=0$. This problem can be resolved by simply using the nominal dissonance value $D_{2 e i}=k_{0} C_{0}$ when $\left(f_{2}-f_{1}\right) / f_{1} \leq$ 0.01 . 
a) $f_{2}>2 f_{1}$ or $\left(f_{2}-f_{1}\right) / f_{1} \leq 0.01$ : Supra-octave or near unison domain.

$$
D_{2 e i}=k_{0} C_{0}
$$

b) $f_{2}-f_{1}<=f_{b}$ : Dynamic domain.

$$
D_{2 e i}=k_{0}\left(100 \frac{2+\log \left(\left(f_{2}-f_{1}\right) / f_{1}\right)}{2+\log \left(f_{b} / f_{1}\right)}+C_{0}\right)
$$

c) $f_{2}-f_{1}>f_{b}$ and $f_{2}<=2 f_{1}$ : Static Domain.

$$
D_{2 e i}=k_{0}\left(90 \frac{\log \left(\left(f_{2}-f_{1}\right) / f_{1}\right)}{\log \left(f_{b} / f_{1}\right)}+10+C_{0}\right)
$$

These equations give the V-curves shown in Fig. 4 of Kameoka \& Kuriyagawa (1969b), reproduced here as Figure 1. $k_{0}$ and $C_{0}$ are scale conversion constants from the relative dissonance scale to the absolute dissonance scale. $D_{2 e i}$ is the dissonance of a dyad assuming components are of equal amplitude at $57 \mathrm{~dB}$ SPL — the actual sound pressure levels are dealt with later.

Kameoka and Kuriyagawa take the above result for $D_{2 e i}$, convert it to an intensity, $D_{I 2 e i}$, subtract the noise, $D_{\text {In }}=C_{0}^{1 / \beta}$, and then convert it back from intensity. $\beta$ is the exponent for the power law which was observed by experiment to be 0.25 . This new $D_{2 e i}$ is then modified according to the sound pressure levels of the two dyadic components, $p_{1}$ and $p_{2}$.

The conversion to dissonance intensity is done by

$$
D_{I 2 e i}=\left(D_{2 e i} / k_{0}\right)^{1 / \beta}
$$

and the intensity of noise is calculated as

$$
D_{\text {In }}=\left(D_{n 0} / k_{0}\right)^{1 / \beta}=C_{0}^{1 / \beta} \quad \text { where } D_{n 0}=k_{0} C_{0}
$$

so the subtraction of noise from the dyad is the new $D_{2 e i}$ :

$$
D_{2 e i}=k_{0}\left(D_{I 2 e i}-D_{I n}\right)^{\beta} .
$$

Kameoka and Kuriyagawa mention (1969b, p. 1463) that when the level difference exceeds about 25 $\mathrm{dB}$ perfect masking occurs and thus no dissonance is added for that dyad $\left(D_{2 i}=0\right)$. The effect of loudness is resolved by relating it to $p_{0}=57 \mathrm{~dB} \mathrm{SPL}=2 \times 10^{-1.15} \mu$ bars. The sound pressure levels are first changed from decibels to linear amplitudes in microbars using the following conversion formula. ${ }^{[3]}$

$$
p_{\mu \mathrm{bar}}=10^{L_{\mathrm{dB}} / 20} / 5000
$$

a) $p_{1}=p_{2}$ : Equal amplitudes.

$$
D_{2 i}=D_{2 e i}\left(p_{1} / p_{0}\right)^{n_{e}}
$$

b) $p_{1}>p_{2}$ : Partial with lower frequency has higher amplitude.

$$
D_{2 i}=D_{2 e i}\left(p_{1} / p_{0}\right)^{n_{e}}\left(p_{2} / p_{1}\right)^{n_{h}}
$$

c) $p_{1}<p_{2}$ : Partial with lower frequency has lower amplitude.

$$
D_{2 i}=D_{2 e i}\left(p_{2} / p_{0}\right)^{n_{e}}\left(p_{1} / p_{2}\right)^{n_{l}}
$$

Kameoka and Kuriyagawa term $D_{2 i}$ the "real absolute dissonance without noise". The exponents for the above equations were determined (Kameoka and Kuriyagawa, 1969a) as $n_{h}=0.15, n_{l}=0.32$, $n_{e}=0.20$. 
Table 1: Spectral Components for Figure 3

\begin{tabular}{rl}
\hline Trial & Spectral Components in $\mathrm{Hz}(d B \mathrm{SPL})$ \\
\hline 1 & $440(60)$ \\
2 & $440(58), 455(50), 484(44), 581(46)$ \\
3 & $440(56), 455(52), 484(52), 880(45)$ \\
4 & $440(57), 484(57)$ \\
5 & $440(54), 455(57), 484(53), 581(54), 880(46)$ \\
6 & $440(50), 455(54), 484(58), 581(55), 880(50)$ \\
7 & $440(45), 455(50), 484(55), 581(58), 880(54)$ \\
8 & $440(42), 455(46), 484(50), 581(55), 880(58)$ \\
9 & $440(50), 484(55), 581(50), 880(58)$ \\
10 & $440(54), 455(43), 484(58), 581(50), 880(59)$ \\
11 & $440(58), 451(50), 484(58), 880(50)$ \\
\hline
\end{tabular}

The final result is once again converted to an intensity $\left(D_{I 2 i}\right)$ and added to the running total of dissonance intensity $D_{I t}$, which is the sum of the dissonance intensities of all combinations of dyads within the spectral makeup of the complex tone plus the ambient noise $D_{I n}$.

$$
\begin{gathered}
D_{I 2 i}=\left(D_{2 i} / k_{0}\right)^{1 / \beta} \\
D_{m}=k_{0}\left(D_{I t}\right)^{\beta}=k_{0}\left(\sum_{i=1}^{m} D_{I 2 i}+D_{I n}\right)^{\beta}
\end{gathered}
$$

$D_{I t}$ is the sum of $D_{I 2 i}$ over all combinations of the spectral components: if there are $n$ partials, then the sum will be over $m=\left(\begin{array}{c}n \\ 2\end{array}\right)=n(n-1) / 2$ dyads (pairs of partials). Once all combinations are summed, the ambient noise $D_{I n}$ is added to give the absolute dissonance $D_{m}$ of the complex tone. The adding and subtracting of noise is done since Kameoka and Kuriyagawa derived their model from experimental data.

\subsection{Implementation under HUMDRUM}

The interpretation of Kameoka and Kuriyagawa's dissonance model described above was implemented in the AWK programming language as a tool called diss, which is designed to work in conjunction with the HUMDRUM Toolkit (Huron, 1995). The diss tool accepts as input a sequence of arbitrary spectra representing the moment-by-moment changes of spectral content such as the successive sonorities in a musical score. diss tool calculates Kameoka and Kuriyagawa's $D_{m}$ measure of total dissonance for each timeslice. The listing for diss appears in the Appendix.

\subsection{Problems with the Model}

Figure 1 is a replica of Kameoka and Kuriyagawa's Figure 4 (1969b), showing that this implementation works well for various frequencies at $57 \mathrm{~dB}$ SPL.

There is marginal discrepancy in the effect of sound pressure level on absolute dissonance between that calculated by Kameoka and Kuriyagawa (1969a, Fig. 9) and this author's implementation, diss. The actual data for their graph was not available and so was estimated from the graph itself.

Other discrepancies appear when diss is used in an attempt to reproduce Figures 5 and 7 from Kameoka and Kuriyagawa (1969b), shown here as Figures 3 and 4. The sound pressure level data for Figure 3 were estimated from Kameoka and Kuriyagawa's Fig. 5 since they did not specify it exactly, which may contribute to some of the discrepancy in the resulting dissonance values. The frequency (amplitude) pairs were estimated as shown in Table 1. This author's implementation of Kameoka and Kuriyagawa's model results in dissonance values that are not as pronounced as those reported in Kameoka and Kuriyagawa's article. Joos 


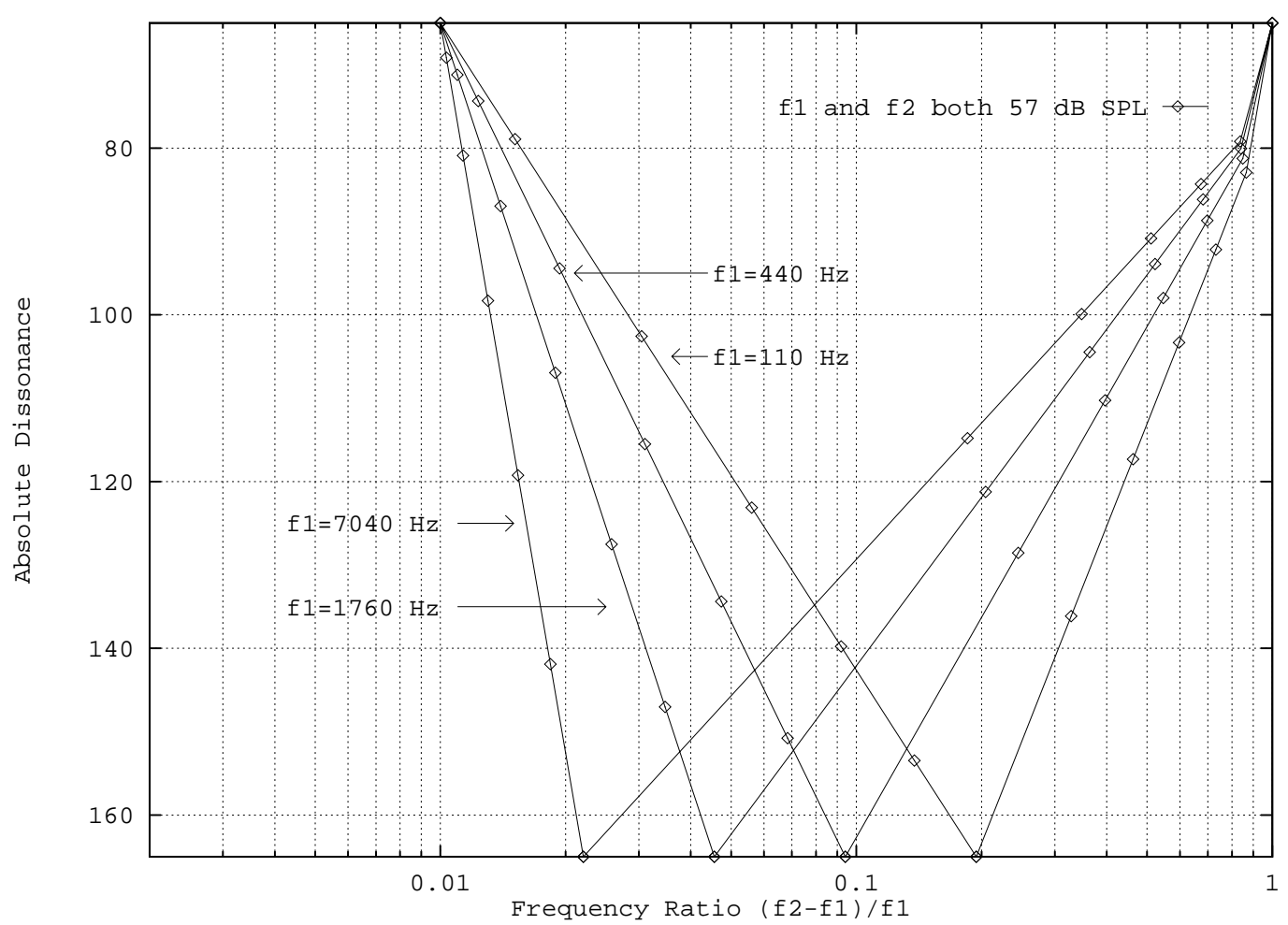

Figure 1: Effect of frequency on dissonance of a dyad of sinusoids (Kameoka \& Kuriyagawa, 1969b, Fig. 4).

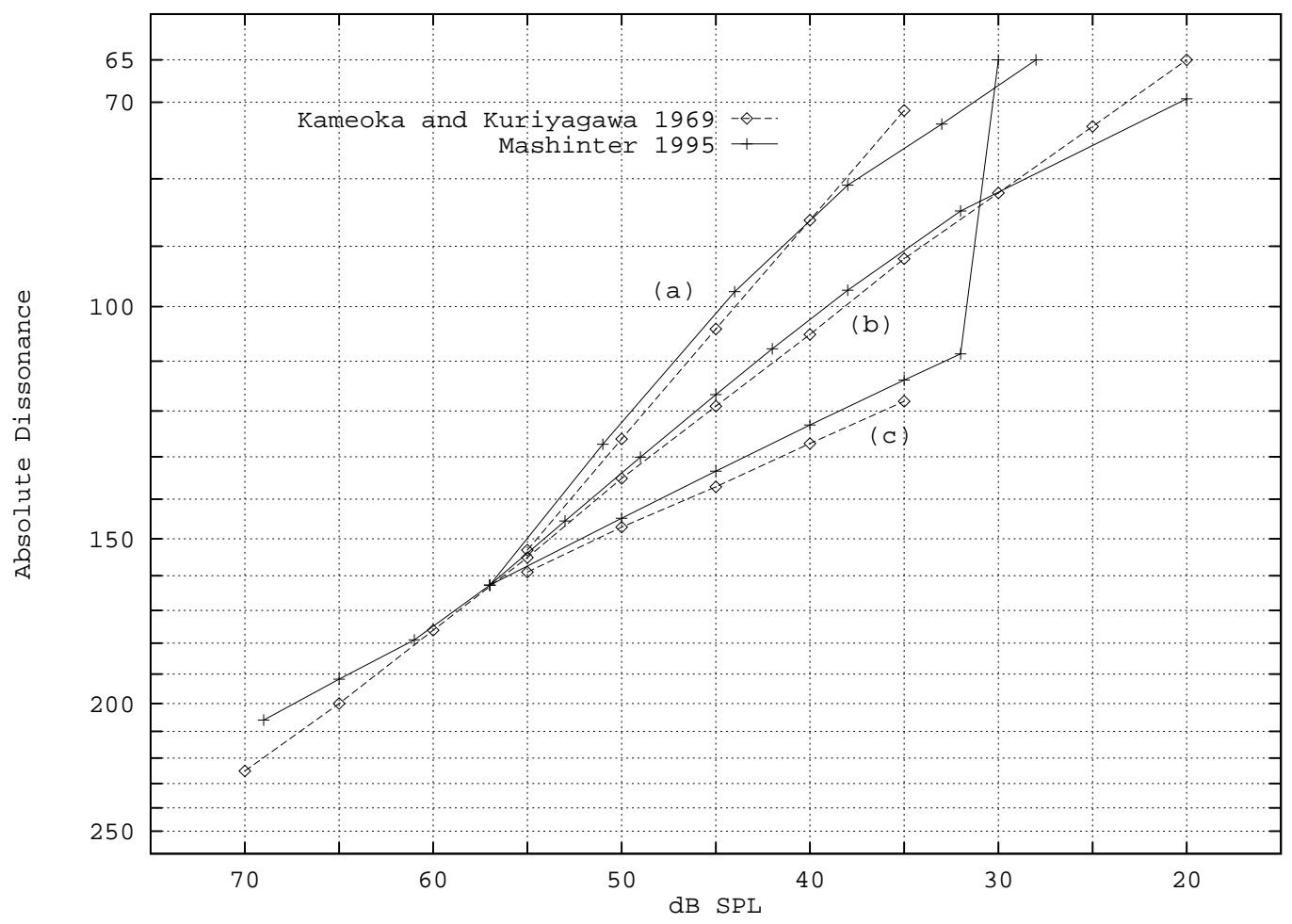

Figure 2: Effect of SPL on dissonance using maximally dissonant dyad $f_{1}=440 \mathrm{~Hz}, f_{2}=484 \mathrm{~Hz}$ (Kameoka \& Kuriyagawa, 1969b, Fig. 9). (a) $L_{1}$ varied while $L_{2}$ held at $57 \mathrm{~dB}$ SPL. (b) $L_{1}$ and $L_{2}$ varied together against reference frequency of $968 \mathrm{~Hz}, 69 \mathrm{~dB}$ SPL. (c) $L_{2}$ varied while $L_{1}$ held at $57 \mathrm{~dB}$ SPL. 


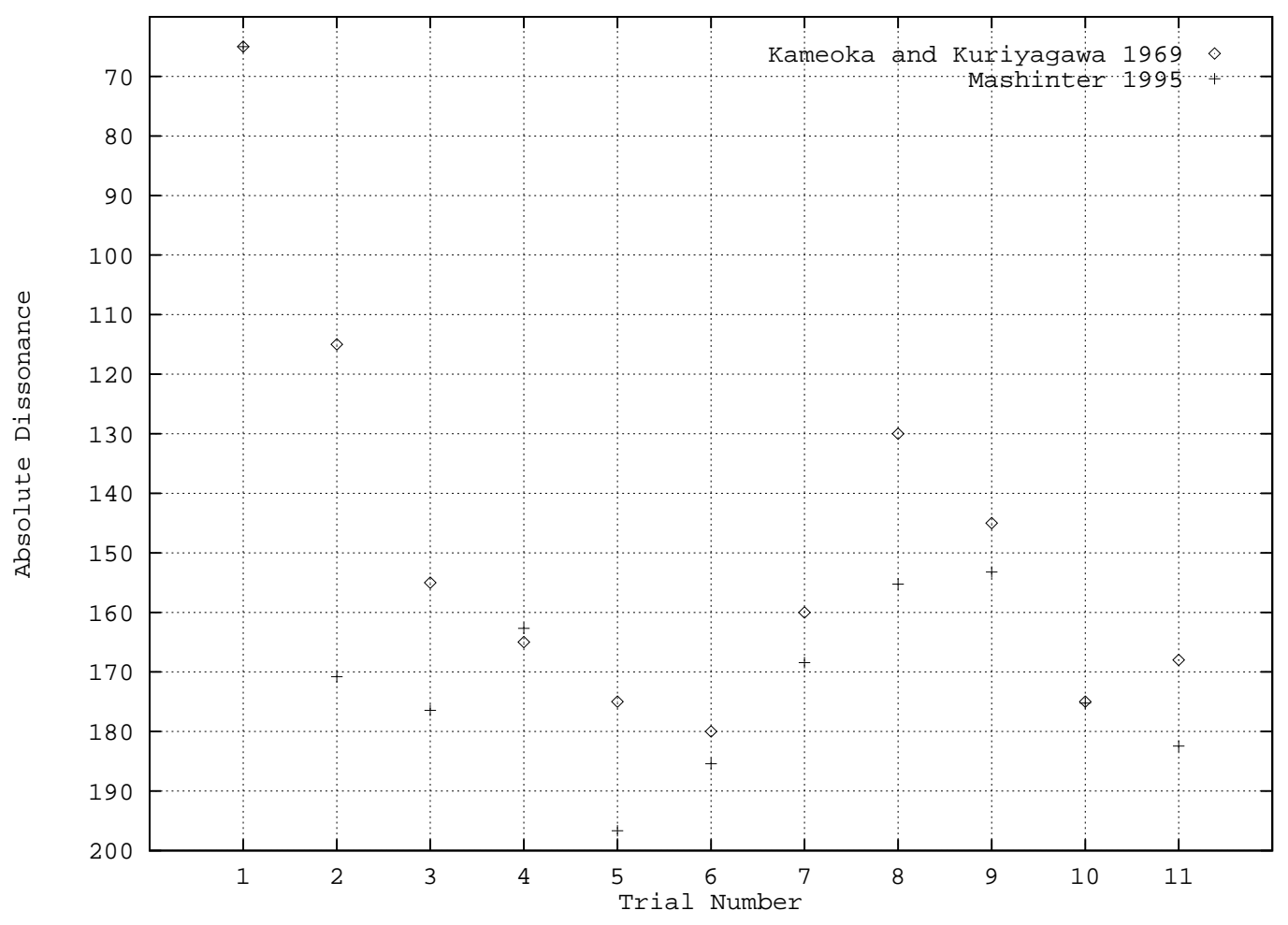

Figure 3: Dissonance of tones of varying complexity (Kameoka and Kuriyagawa, 1969b, Fig. 5).

Vos (1986) also observed this discrepancy when comparing his tuning purity ratings with absolute dissonance predictions. These results suggest that there is likely a problem with Kameoka and Kuriyagawa's procedure or results.

Another problem with the model is that the absolute dissonance increases monotonically with the number of partials. As partials are added to a sound, dissonance necessarily increased. That is, the more partials you have in a sonority, the more dissonant the sound is. David Huron has noted, however, that this is inconsistent with some commonplace musical perceptions. For example, most listeners will perceive a major-major seventh chord as less dissonant than an open seventh interval (e.g., C-E-G-B vs. C-B). That is, adding the pitches $\mathrm{E}$ and $\mathrm{G}$ tends to reduce the perceived dissonance of the bare seventh formed by the dyad $\mathrm{C}$ and $\mathrm{B}$. Although this example may involve some element of enculturation or learning, the general principle still holds.

\subsection{Vos's Critique of Kameoka and Kuriyagawa's Model}

Vos (1986) compared his experimental data for detecting dissonance minima (maximal tuning) with dissonance patterns predicted by the models of Plomp \& Levelt and Kameoka \& Kuriyagawa. As more harmonics are considered in either Kameoka \& Kuriyagawa's model or Hutchinson \& Knopoff's, the differences in predicted dissonance between different pairs of complex tones—say a minor second and major fifth—decrease considerably, often making the predictions unreasonable.

Vos was particularly interested in the sharpness of the peaks of predicted dissonance as they relate to tuning. He found that predictions from Plomp and Levelt's model conditionally agree with his purity ratings, but Kameoka and Kuriyagawa's model is less agreeable. The results for both models are blurred as more harmonics are considered. In general, variance decreases when more data are considered, but Vos observed that Kameoka and Kuriyagawa's model is quite at odds with his tuning ratings. In one case, Kameoka and Kuriyagawa's model predicts that the dissonance for a justly tuned fifth (2:3) would actually sound more dissonant than surrounding detunings.

Vos also pointed out that Kameoka and Kuriyagawa's model does not produce as much variance as 


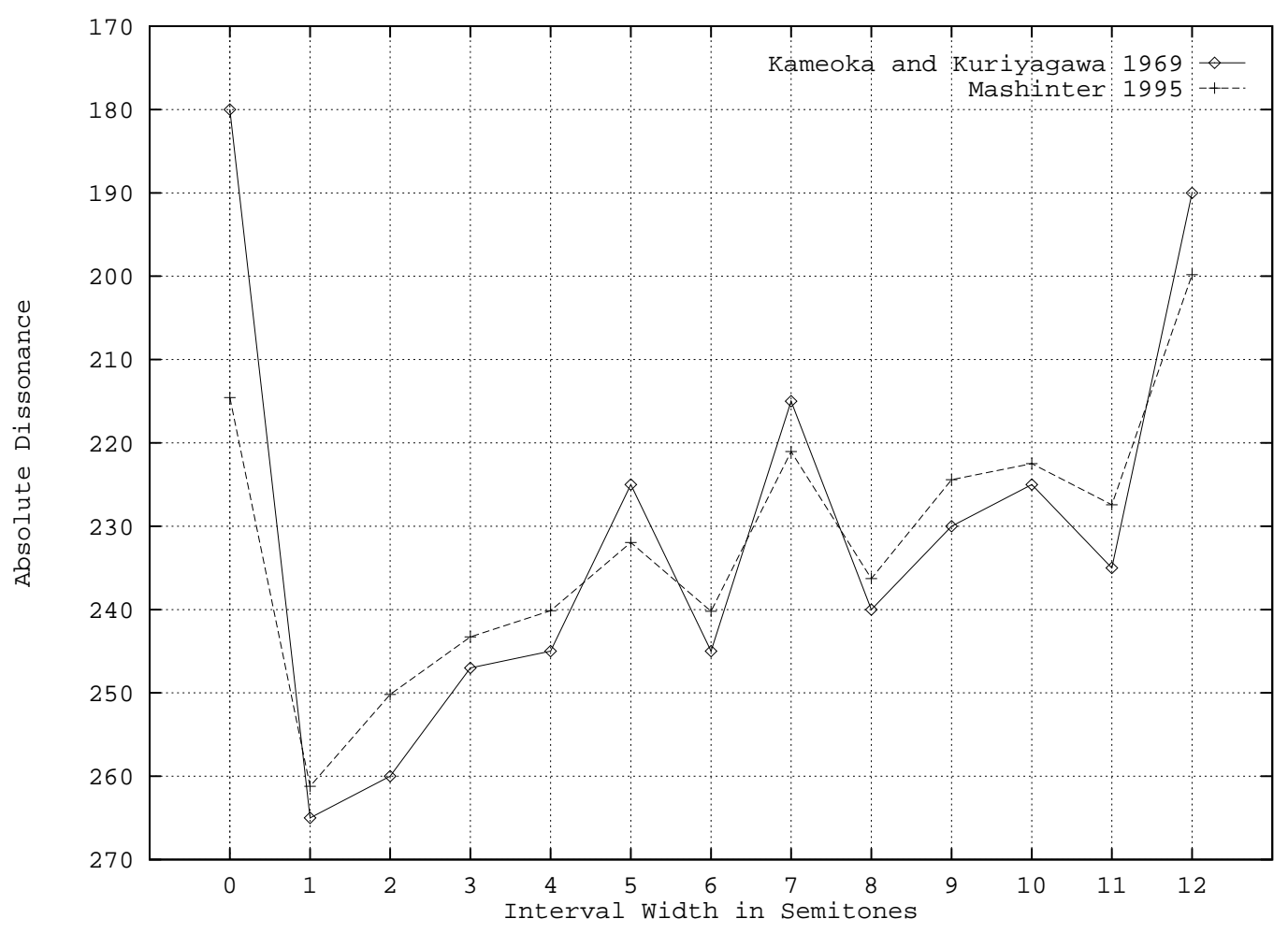

Figure 4: Dissonance by interval between two identical complex tones with the following harmonic structure: 1st harmonic (-12 dB), 2(0), 3(0), 4(-4), 5(-8), 6(-12), 7(-14), 8(-16) (Kameoka \& Kuriyagawa, 1969b, Fig. 7).

their published results indicate. This author's implementation supports Vos (Figure 4), indicating a problem with Kameoka and Kuriyagawa's method or results. This author agrees with Vos's complaint that Kameoka and Kuriyagwa's treatment of background noise and scaling is ill-devised-as more harmonics are added, the difference in dissonance ebbs away between different intervals. That is, for tones of increasing complexity, the difference in "absolute dissonance" between, say, a minor second and a perfect fifth becomes smaller. This is a logical phenomena, but Kameoka and Kuriyagawa's scale appears to overrate it. When ambient noise is discounted, the problem worsens further (Vos, 1986, p. 255).

Kameoka and Kuriyagawa use the power function for adding all dissonances, but Vos notes this is inconsistent with Zwicker's (1957) model of loudness summation. Zwicker only sums with the power function when the frequencies of partials lie in the same critical band. In different critical bands, Zwicker proposes an arithmetic summation similar to that used by Plomp and Levelt (1965). Perhaps a marriage of some of Kameoka and Kuriyagawa's methods with Plomp and Levelt's would yield a more durable model for sensory dissonance, but for now we move on to consider Hutchinson and Knopoff's dissonance-a model which has problems of its own in both theory and implementation.

\section{Hutchinson AND KNOPOFF}

William Hutchinson and Leon Knopoff (1978) proposed a dissonance model for dyads; they subsequently extended their model to include dissonance estimations for three- and four-sonorities (1979). Their work is based on Plomp and Levelt's (1965) observations relating dissonance to critical bandwidth, but they empirically derive their own curve for critical bandwidth itself. 
Their model for total dissonance is as follows:

$$
D=\frac{\frac{1}{2} \sum_{i=1}^{N} \sum_{j=1}^{N} A_{i} A_{j} g_{i j}}{\sum_{i=1}^{N} A_{i}^{2}}=\frac{\sum_{i=1}^{N} \sum_{j=i+1}^{N} A_{i} A_{j} g_{i j}}{\sum_{i=1}^{N} A_{i}^{2}}
$$

where $N$ is the number of partials, $A_{i}$ are the amplitudes of the partials, and $g_{i j}$ is the dissonance of a dyad based on the frequency components and critical bandwidth. Hutchinson and Knopoff fit the critical band width CBW empirically, based on data from Cross and Goodwin, and from Mayer ${ }^{[4]}$ :

$$
\mathrm{CBW}=1.72(\bar{f})^{0.65} \quad \text { where } \bar{f}=\left(f_{1}+f_{2}\right) / 2
$$

The dissonance factor $g$ is a function of $y$, the CB interval:

$$
g=g(y), \quad y=\left|f_{2}-f_{1}\right| / \mathrm{CBW}
$$

Richard Parncutt ${ }^{[5]}$ devised a function that well represents Plomp and Levelt's dissonance factor $g$ as shown below. The resulting implementation of Hutchinson and Knopoff's model is included in the Appendix as rough. The author's implementation eliminates the problems associated with shifting harmonic frequencies to their nearest equally tempered pitches, using the solution described below.

$$
g(y)=\left(\frac{y}{a} \exp ^{\left(1-\frac{y}{a}\right)}\right)^{b} \quad a=.25, b=2
$$

\subsection{Problems with the Model}

Parncutt, as in Hutchinson and Knopoff (1978, p. 7), shifts the frequencies of the overtones to frequencies coinciding with the nearest equally-tempered pitch. As frequencies rise, the difference between pitch frequencies increases, augmenting the possible error between harmonic frequencies and their nearest pitches. This could cause large deviations in the results, but the amplitude of harmonics for "natural" tones usually decreases with increasing harmonic number. Thus, higher frequencies will contribute less to the overall dissonance since their amplitude is lower. Assuming that the amplitude of the $n^{\text {th }}$ harmonic varies as $1 / n$, the relative error is less than $1 \%$.

Table 2 shows the average error in the CB interval, its variance, and the total error in dissonance due to shifting the frequencies of harmonics to their nearest equal-tempered pitches. ${ }^{[6]}$

Table 2: Average error in CB Interval due to Shifting Harmonics

\begin{tabular}{clll}
\hline Fundamental & Average Error & Variance & Error in $g$ \\
\hline C1 to B1 & 0.00245 & 0.0000186 & 0.000696 \\
C2 to B2 & 0.00312 & 0.0000302 & 0.00112 \\
C3 to B3 & 0.00398 & 0.0000490 & 0.00181 \\
C4 to B4 & 0.00507 & 0.0000796 & 0.00292 \\
C5 to B5 & 0.00647 & 0.000129 & 0.00469 \\
C6 to B6 & 0.00824 & 0.000210 & 0.00752 \\
\hline
\end{tabular}

Hutchinson and Knopoff shift harmonics to their closest equal temperament pitch, introducing error into the calculation of CB interval $y=\left|f_{2}-f_{1}\right| / C B W$ and dissonance factor $g=g(y)$ as shown. The timbres used were weighted in amplitude as $1 / n$ for $n$ up to 10 harmonics.

Hutchinson and Knopoff (1978, p. 8) incorrectly claim that "the use of well-tempered pitches for the overtones instead of the just temperament pitches produces no significant errors because of the smoothness of the [Plomp \& Levelt dissonance factor] curve,". The dissonance curve has a steep slope, meaning that small changes in the critical band interval can create large differences in the dissonance factor. That is, errors 


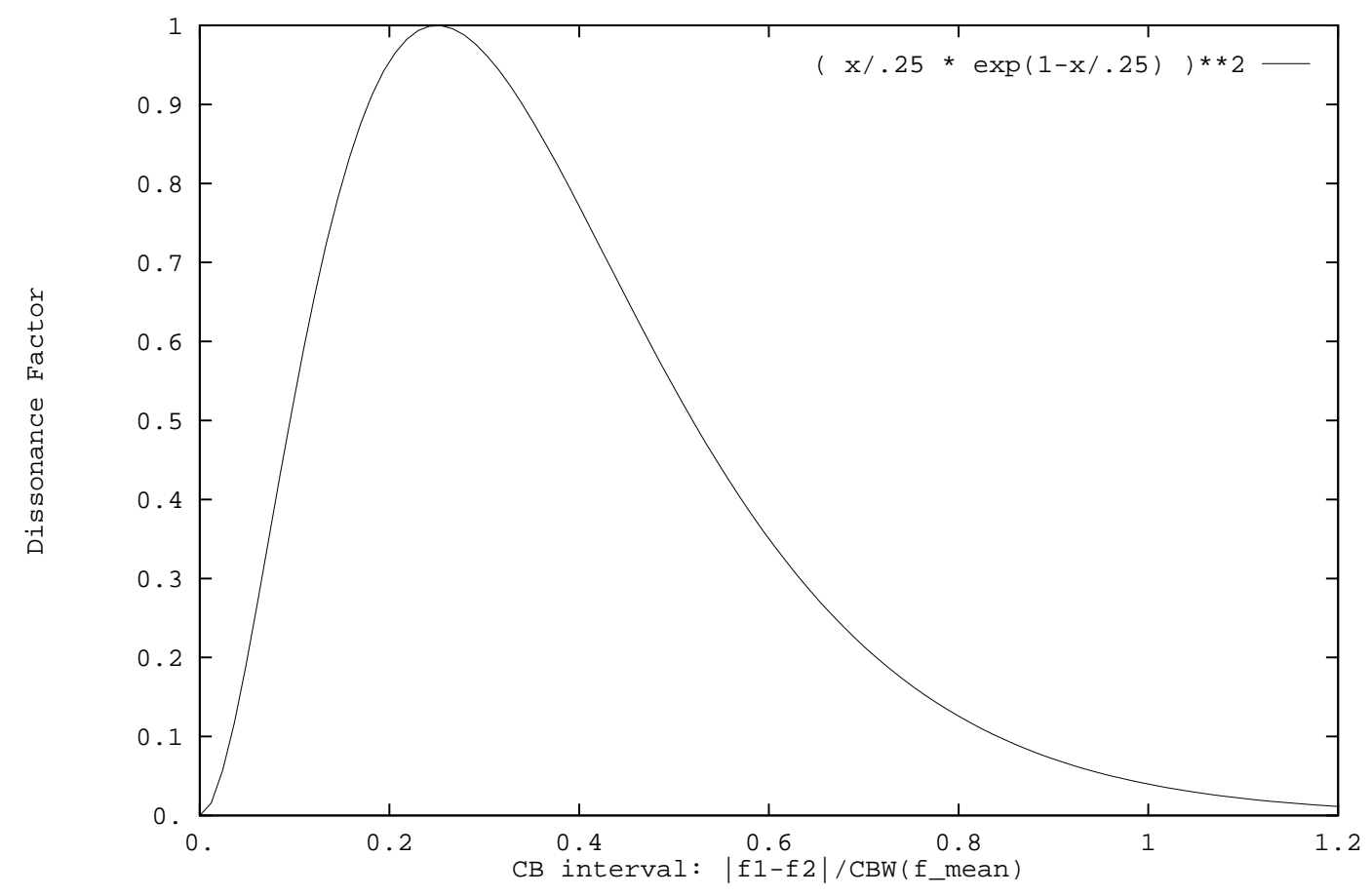

Figure 5: Parncutt's approximation to Plomp and Levelt's dissonance factor $g$.

are amplified, not reduced. The real reason for the dissipation of error is due to the $1 / n$ weighted amplitude timbres that they use, as noted above.

Figure 5 shows Parncutt's approximation to Plomp and Levelt's dissonance factor $g$ which peaks at .25 of the CB interval. Figure 6 shows the difference in $\mathrm{CB}$ interval when harmonic frequencies are shifted to the nearest pitch. The curves from lowest to highest represent equal-amplitude harmonics of tones with base frequencies C2, C3, C4, C5, and C6. At the fifth harmonic, the error in the CB interval approaches 0.1, which translates to a dissonance factor error of about $50 \%$. The seventh harmonic produces even worse error: almost .25 of the CB interval or almost $100 \%$ error in the dissonance factor. Granted, this error is typically reduced for higher partials, but shifting harmonics to their nearest equal-temperament pitch only introduces error. The computational savings of such shifts is modest compared with the errors introduced. To ensure that sound pressure levels of the same frequency are summed, the partials can be sorted in order of frequency, and adjacent partials of the same frequency can then be combined.

\section{Sample Results from the Models}

Neither Kameoka and Kuriyagawa's model nor Hutchinson and Knopoff's produces the results that they publish. The author's implementation of Hutchinson and Knopoff's model, rough, produces data lower than published results (Hutchinson \& Knopoff, 1978, 1979), likely due to Parncutt's approximation to Plomp and Levelt's dissonance factor and the use of true frequency multiples for harmonics rather than approximate equally-tempered pitches. The considerable number of calculations may also introduce round-off error. The problems with Kameoka and Kuriyagawa's model, implemented in this research as diss, were described above. Some results for each model are shown in Table 3 for comparison. 


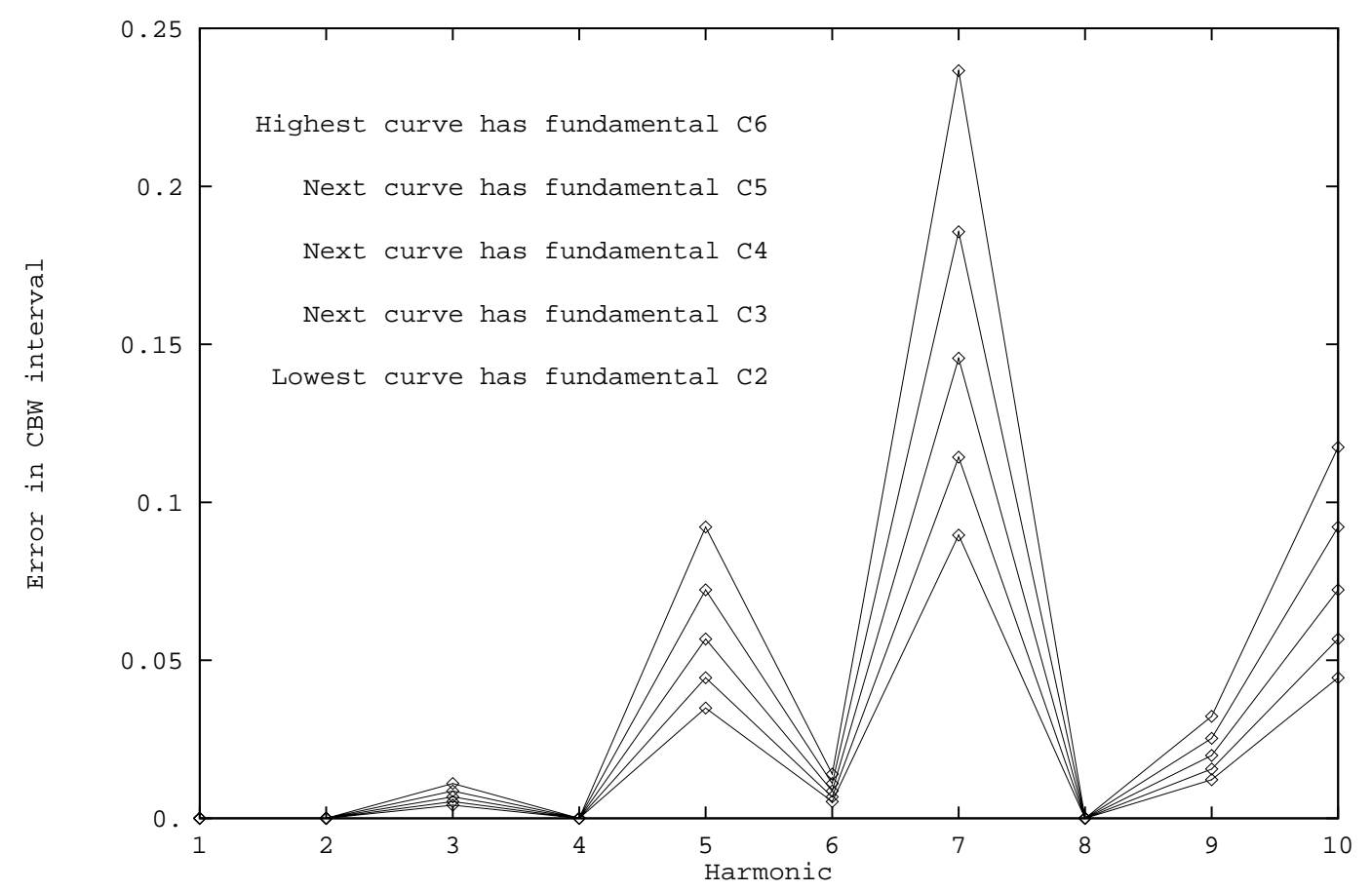

Figure 6: Error in $\mathrm{CB}$ interval due to shifting frequencies of harmonics to the nearest pitch.

\section{Conclusions}

Some forty-five years have elapsed since Donald Greenwood's seminal publications (1961a; 1961b) relating sensory dissonance to physiological properties of the hearing organ. Sensory dissonance remains an elusive phenomenon, but at least some basic principles appear to be uncontentious. These principles are summarized below along with some practical observations related to developing models for dissonance.

\subsection{Principles of Sensory Dissonance}

1. Sensory dissonance depends on timbre. More specifically, it depends on the amount of interaction between pairs of partials, or dyads, of which the sound is composed.

2. Sensory dissonance increases with loudness. This is partly due to increased critical bandwidth, but more study is need to refine this relationship.

3. For two pure tones, the frequency difference for maximum dissonance increases with mean frequency, but not logarithmically (with pitch). The dissonance of a major third, for example, depends on tessitura. This frequency difference increases with the size of critical bands through increases in frequency.

4. Dissonance is relative to its context. For example, a bare seventh (C-B) may be considered more dissonant than a full seventh chord (C-E-G-B). ${ }^{[7]}$

\subsection{Practical Matters of Dissonance}

1. Functions that model dissonance based on frequency differences and critical bands are idealized for "normal hearing", and ignore possible cultural differences. Further, it is important to consider the difference between non-musician and musician subjects. Musicians' judgments may be influenced by preconceived theoretical notions of dissonance. In addition, little attention has been paid to the 
Table 3: Comparison of Results from diss and rough

\begin{tabular}{llll}
\hline Harmonic & Equal-tempered & \multicolumn{2}{c}{ Results } \\
\cline { 3 - 4 } Structure & Pitches & 227.87 & 0.00002 \\
\hline unison & c c & 265.71 & 0.4779 \\
m2 & c db & 264.78 & 0.2185 \\
M2 & c d & 258.00 & 0.0923 \\
m3 & c eb & 253.00 & 0.0670 \\
M3 & c e & 248.13 & 0.0516 \\
P4 & c f & 248.07 & 0.1373 \\
tritone & c gb & 237.01 & 0.0219 \\
P5 & c g & 243.69 & 0.1342 \\
m6 & c ab & 239.10 & 0.0685 \\
M6 & c a & 241.29 & 0.1201 \\
m7 & c bb & 243.05 & 0.2791 \\
M7 & c b & 310.94 & 0.1657 \\
linv major & d f bb & 307.85 & 0.1166 \\
2inv major & c f a & 308.00 & 0.1174 \\
linv minor & c e a & 306.10 & 0.1401 \\
2inv minor & d f b & 312.08 & 0.1724 \\
sus2 triad & d e a & 310.28 & 0.1455 \\
sus4 triad & d g a & 310.63 & 0.1041 \\
minor triad & d f a & 309.92 & 0.0967 \\
major triad & d f $\sharp$ a & 317.99 & 0.1878 \\
diminished triad & d f ab & 358.77 & 0.2538 \\
M7 chord & c e g b & 363.19 & 0.2331 \\
m7 chord & c e g bb & 361.29 & 0.3131 \\
mM7 chord & c eb g b & 362.67 & 0.2378 \\
half-dim7 chord & c e g b & 366.85 & 0.2718 \\
dim7 chord & c $\sharp$ e g bb & & \\
\hline
\end{tabular}

The timbre uses harmonic amplitudes $1 / n$ for $n$ up to 10 harmonics. All fundamental pitches are equally-tempered in the range $[\mathrm{C} 4, \mathrm{C} 5]$.

dynamic aspects of tone. Most studies consider timbres as static, but modern cochlear models and Fourier transforms make it possible to study the dynamic aspect as well (Simpson, 1994).

2. Kameoka and Kuriyagawa's experiments indicate a relationship between dissonance and masking: both have a stronger and wider effect for higher frequency components since higher frequency components stimulate more of the basilar membrane and auditory nerve than lower frequency components (see Pickles, 1988 , pp. $38-52$ on the cochlea, pp. 88-89 on the auditory nerve, and pp. $258-260$ for loudness/masking relationship). This point may serve to reinforce or redefine the relationship between dissonance, loudness, and masking.

3. Kameoka and Kuriyagawa's experiments also show that the frequency difference of maximal dissonance increases not only with mean frequency, but also with sound pressure level, the effect of which has not yet been fully recognized and studied. Glasberg and Moore (1990) still claim that level differences have no appreciable effect on auditory dissonance, and many researchers still refer to the critical bandwidth curve proposed by Zwicker, Flottorp, and Stevens (1957) although other representations, particularly Greenwood's (1961a; 1961b), have proven better.

4. The additivity of dissonance is based on conjecture in both Kameoka \& Kuriyagawa and Hutchinson and Knopoff. The former use psychological weighting whereas the latter average a squared sum. The additivity of dissonance is a critical point in the construction of a dissonance model for complex tones, but the existing methods do not satisfy all the axioms noted above.

5. The effect of tonal fusion needs to be distinguished from consonance or and dissonance in a model for the latter. Tonal fusion is the tendency for two tones with highly coincident partials as one tone, which can be a disconcerting sensation. Existing evidence already shows that in polyphonic compositions, consonant intervals that promote tonal fusion are treated differently than consonant intervals that have 
low tonal fusion. Huron (1991) showed that Bach tends to avoid tonal fusion while pursuing tonal consonance—-frequent octaves, fifths, and fourths are avoided while thirds and sixths are pursued.

6. The true frequencies of harmonics should not be approximated by the nearest pitch frequency. Summing frequencies of the same amplitude can be done after first sorting the partials by frequency.

Hopefully this discussion has exposed some of the pitfalls in existing models of sensory dissonance so that future research can seek a more productive path. ${ }^{[8]}$ 


\section{Notes}

${ }^{[1]}$ This research was originally produced for an honors undergraduate thesis in Applied Mathematics and Music at the University of Waterloo, 1995.

${ }^{\text {[2] }} 1 \mathrm{bar}=10^{5} \mathrm{~Pa}$, so $1 \mu \mathrm{bar}=0.1 \mathrm{~Pa}$. The lowest sounds pressure detectable by most human ears is $2 \times$ $10^{-5} \mathrm{~N} / \mathrm{m}^{2}=20 \mu \mathrm{Pa}$ RMS. Intensity level in $\mathrm{dB} \mathrm{SPL}=20 \log _{10}\left(\frac{R M S \text { sound pressure }}{2 \times 10^{-5} \mathrm{~N} / \mathrm{m}^{2}}\right)$.

[3] The author thanks Dr. John Vanderkooy at the Physics Department of the University of Waterloo for his assistance in determining this conversion. Kameoka and Kuriyagawa are somewhat vague on this point.

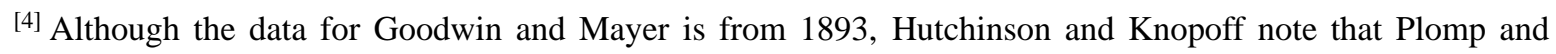
Levelt's CBW data is relatively ambiguous for low pitched sounds (Hutchinson \& Knopoff, 1978, p. 5).

${ }^{[5]}$ Richard Parncutt graciously provided a sample algorithm after Hutchinson and Knopoff (1978) which was revised and enhanced.

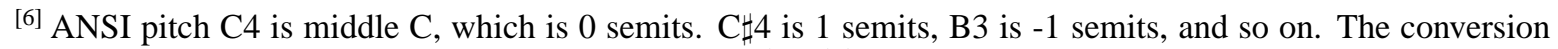
from semits $S$ to frequency $f$ in $\mathrm{Hz}$ is $f=440 \times 2^{(S-9) / 12}$.

[7] The importance of context was emphasized by Huron.

${ }^{[8]}$ Many thanks are extended to Dr. David Huron for his encouragement and support through the frustrations of this research. Originally, we had sought to test the hypothesis that composers tend to place consonant sonorities on strong beats but were waylayed by difficulties with these models of dissonance.

\section{REFERENCES}

Bregman, A. B. (1990). Auditory scene analysis: the perceptual organization of sound. Cambridge: MIT Press.

Cazden, N. (1980). “The definition of consonance and dissonance." International Review of the Aesthetics and Sociology of Music. Vol. 2, pp. 123-168.

Glasberg, B. R. and Moore, B. C. J. (1990). "Derivation of auditory filter shapes from notched-noise data." Hearing Research. Vol. 47, pp. 103-138.

Greenwood, D. D. (1961a). "Auditory masking and the critical band.” Journal of the Acoustical Society of America. Vol. 33, pp. 484-501.

Greenwood, D. D. (1961b). "Critical bandwidth and the frequency coordinates of the basilar membrane." Journal of the Acoustical Society of America. Vol. 33, pp. 1344-1356.

Greenwood, D. D. (1991). "Critical bandwidth and consonance in relation to cochlear frequency-position coordinates." Journal of the Acoustical Society of America. Vol. 54, pp. 64-208.

Huron, D. (1991). “Tonal Consonance versus Tonal Fusion in Polyphonic Sonorities.” Music Perception. Vol. 9, No. 2, pp. 135-154.

Huron, D. (1995). "The Humdrum Toolkit: Reference Manual” Menlo Park, California: Center for Computer Assisted Research in the Humanities. ISBN 0-936943-10-6.

Huron, D. and Sellmer, P. (1992). "Critical Bands and the Spelling of Vertical Sonorities." Music Perception. Vol. 10, No. 2, pp. 129-149. 
Hutchinson, W. and Knopoff, L. (1978). “The Acoustic Component of Western Consonance.” Interface. Vol. 7, No. 1, pp. 1-29.

Hutchinson, W. and Knopoff, L. (1979). "The significance of the acoustic component of consonance in Western triads.” Journal of Musicological Research. Vol. 3, pp. 5-22.

Kameoka, A. and Kuriyagawa, M. (1969a). "Consonance theory, part I: Consonance of dyads." Journal of the Acoustical Society of America. Vol. 45, No. 6, pp. 1451-1459.

Kameoka, A. and M. Kuriyagawa. (1969b). “Consonance theory, part II: Consonance of complex tones and its computation method.” Journal of the Acoustical Society of America. Vol. 45, No. 6, pp. 1460-1469.

Parncutt, R. (1989). Harmony: A Psychoacoustical Approach. Springer-Verlag.

Pickles, J. O. An Introduction to the Physiology of Hearing. 2nd ed. London: Academic Press.

Plomp, R. and Levelt, W. J. M. (1965). “Tonal Consonance and Critical Bandwidth.” Journal of the Acoustical Society of America. Vol. 38, pp. 548-560.

Simpson, J. (1994). “Cochlear Modeling of Sensory Dissonance and Chord Roots.” Master's Thesis, Systems Design Engineering. University of Waterloo.

Tenney, J. (1988). A History of Consonance and Dissonance. New York: Excelsior.

Vos, J. (1986). "Purity Ratings of Tempered Fifths and Major Thirds." Music Perception. Vol. 3, No. 3, pp. $221-258$.

Yost, W. A. and Nielsen, D. W. (1977). Fundamentals of Hearing: An Introduction. New York: Holt, Rinehart, and Winston.

Zwicker, E., Flottorp, G., and Stevens, S. S. (1957). "Critical bandwidth in loudness summation." Journal of the Acoustical Society of America. Vol. 29, pp. 548-557.

Zwicker, E. (1974). “On a psychoacoustical equivalent of tuning curves.” Facts and Models in Hearing. Berlin: Springer. pp. 132-141.

\section{APPENDIX}

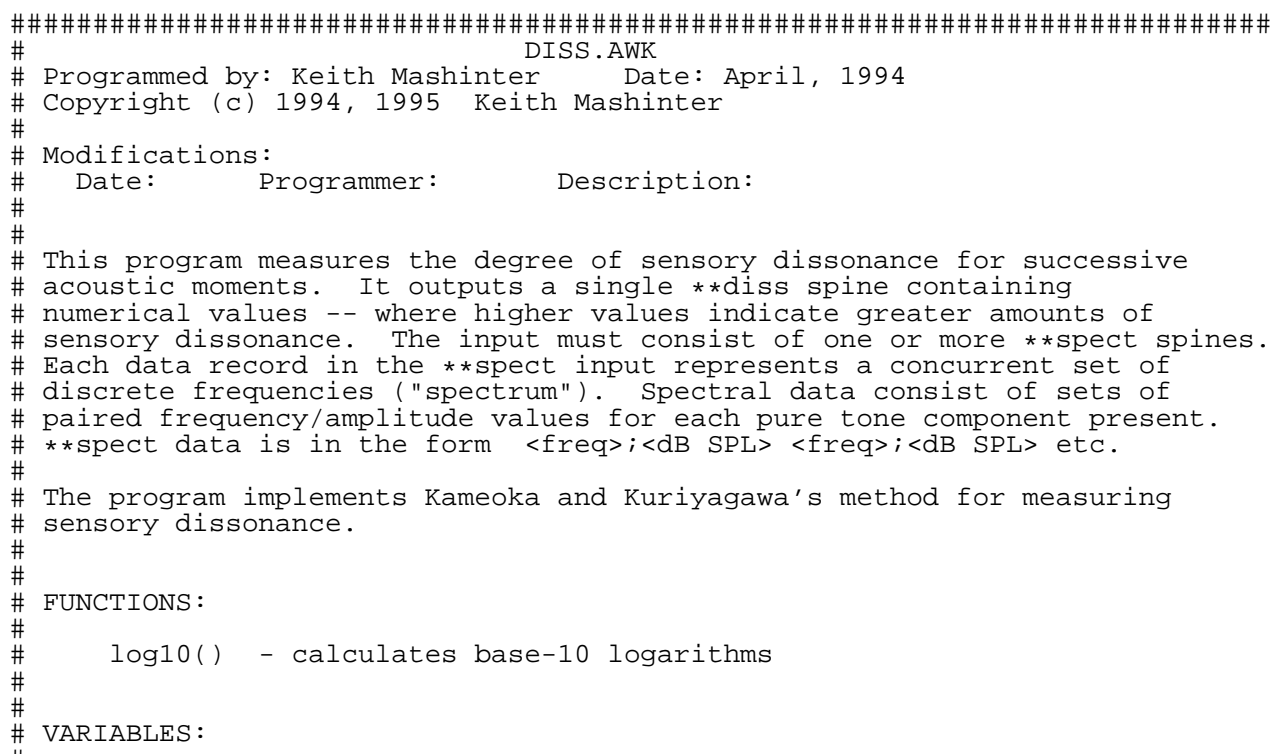




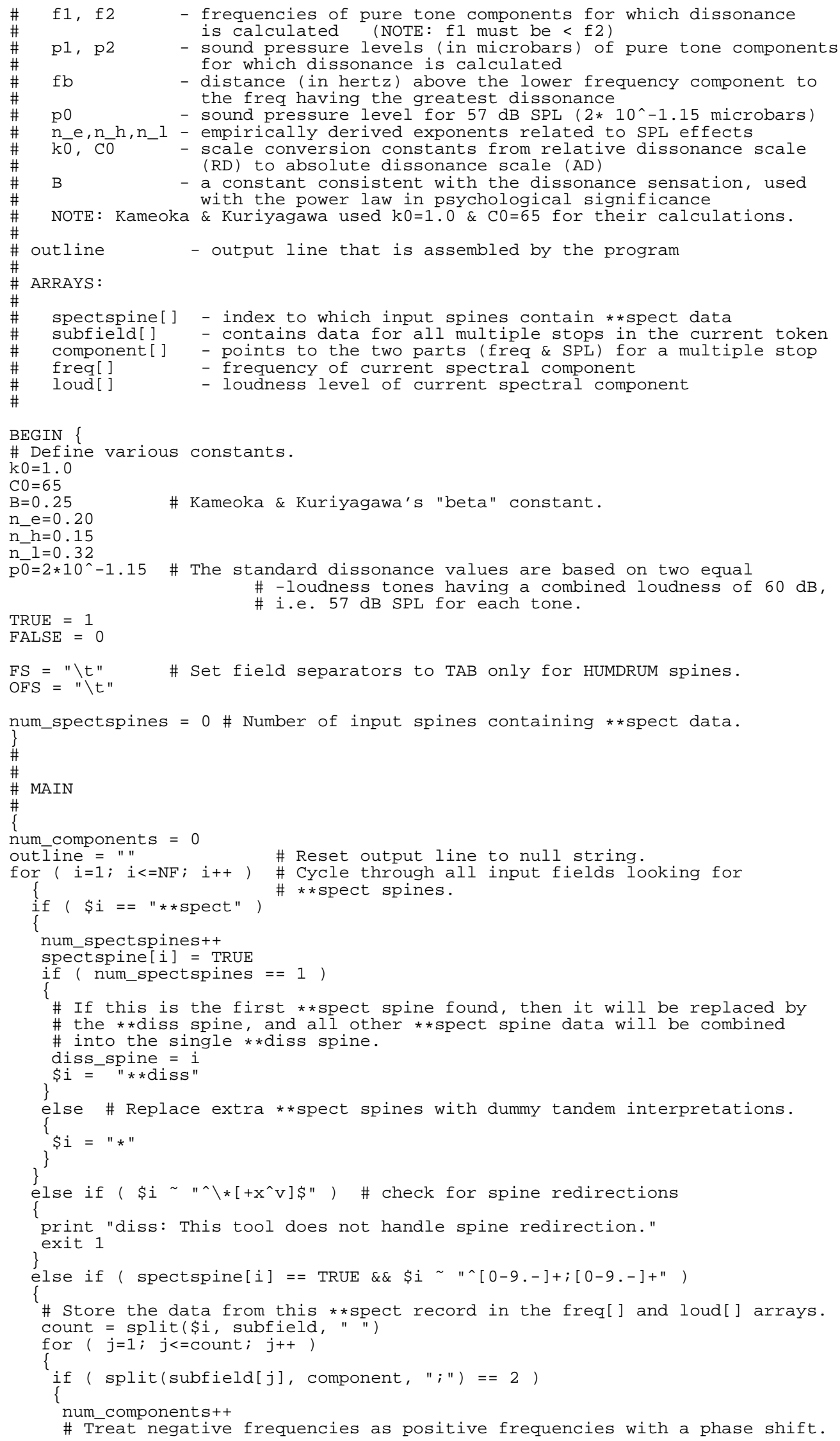



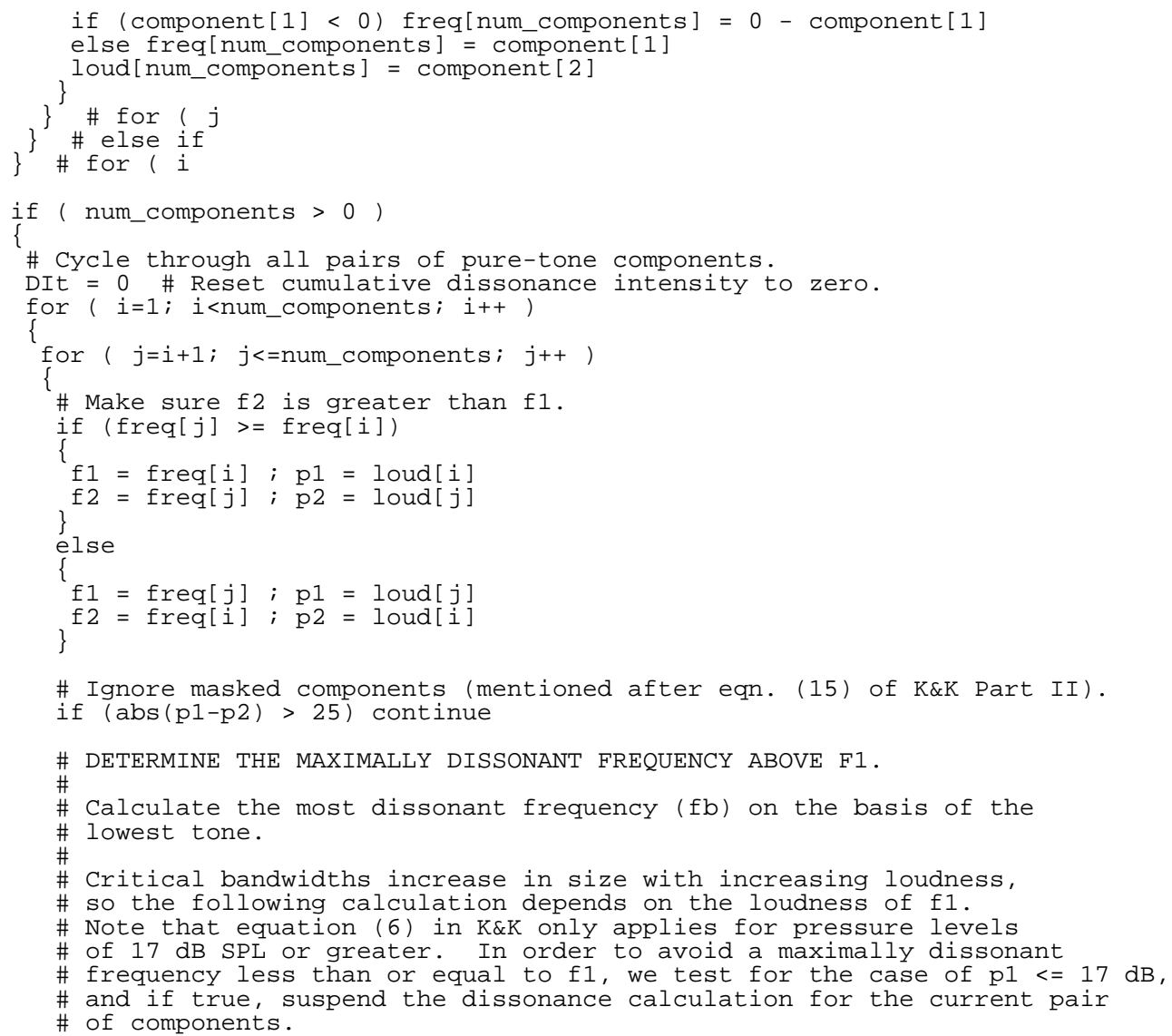

if ( $1<=17)$ continue

$\mathrm{fb}=2.27 \star(((\mathrm{p} 1-57) / 40)+1) \star \mathrm{f} 1 \wedge 0.477$ \# Equation (6).

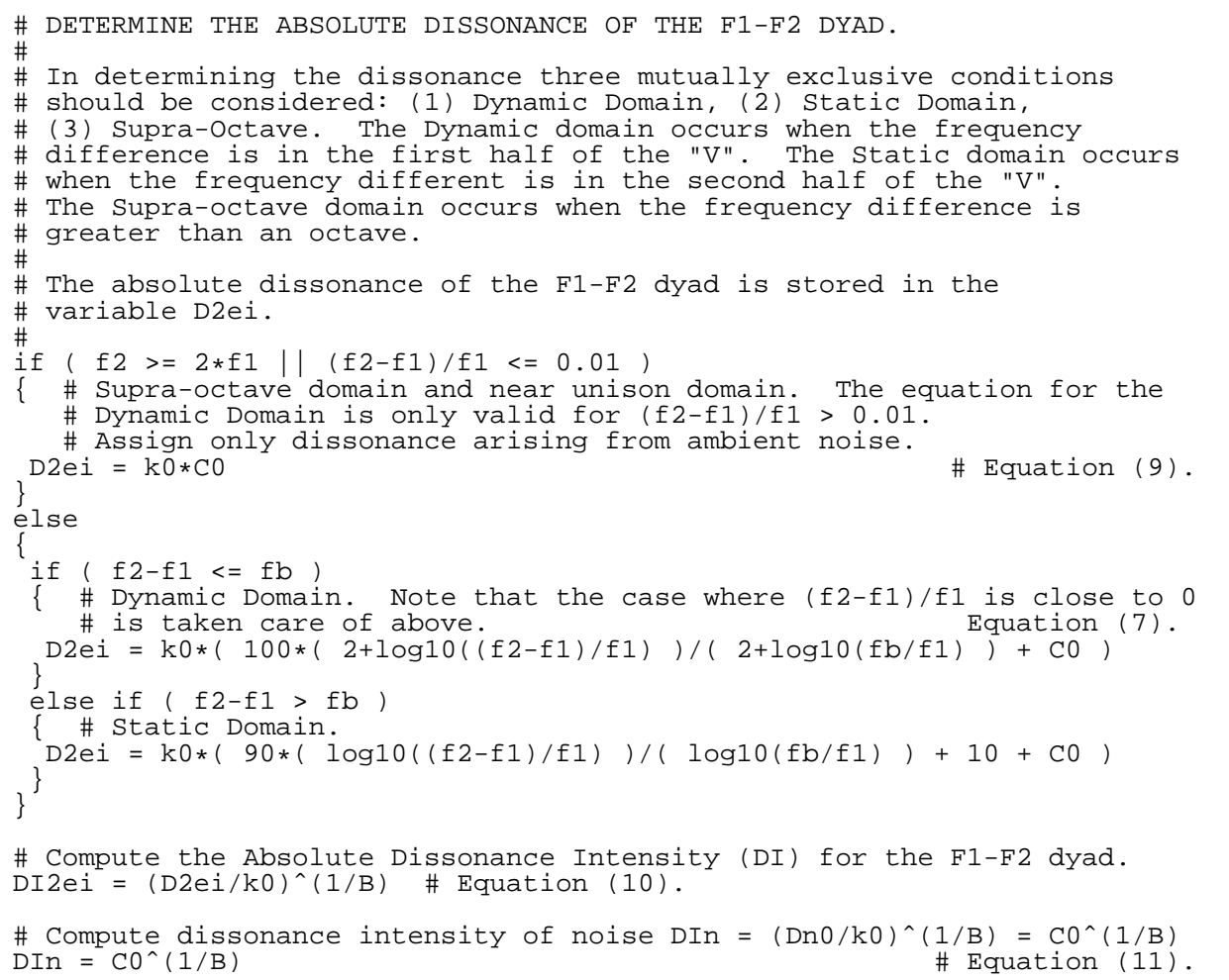




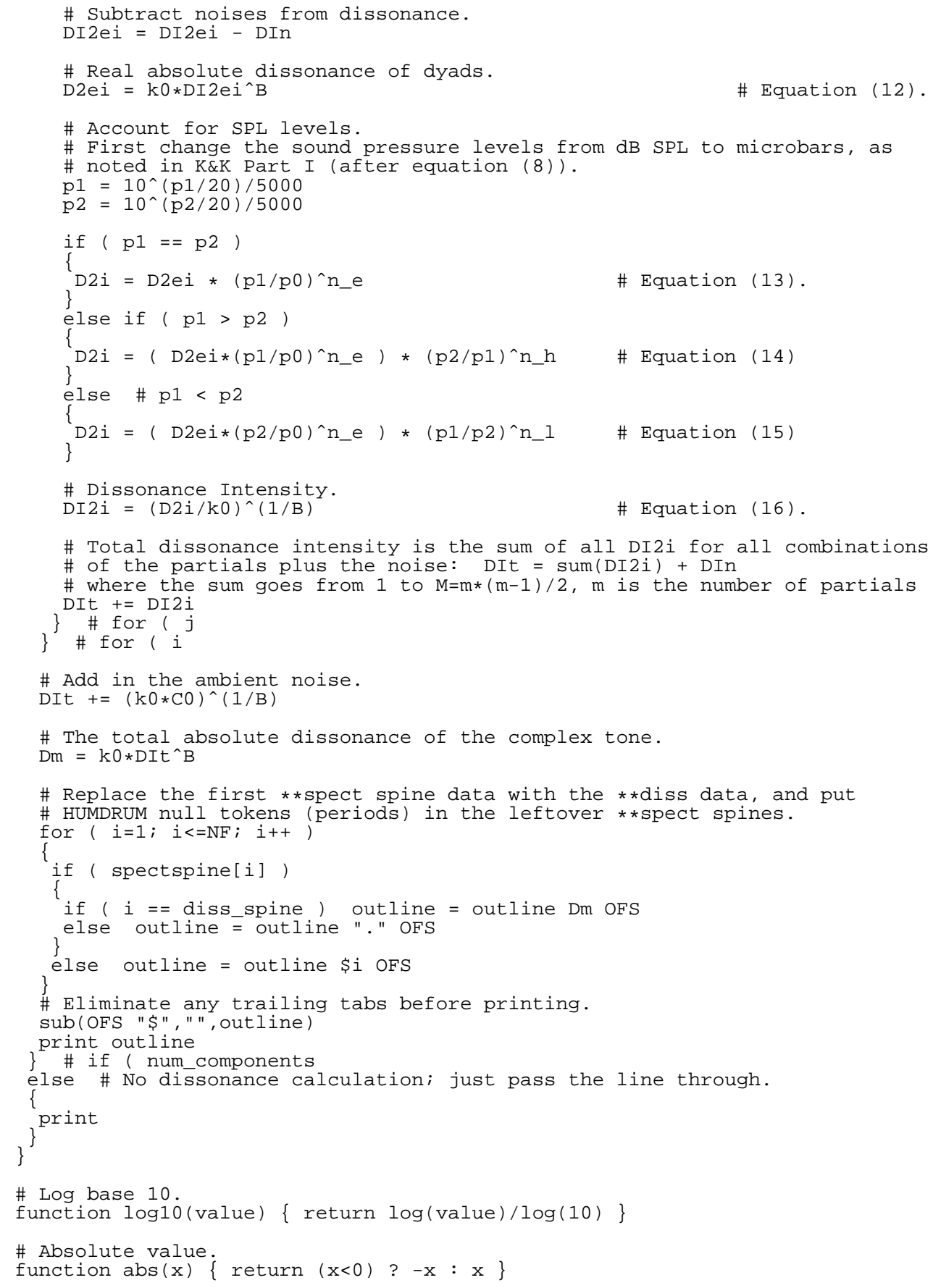




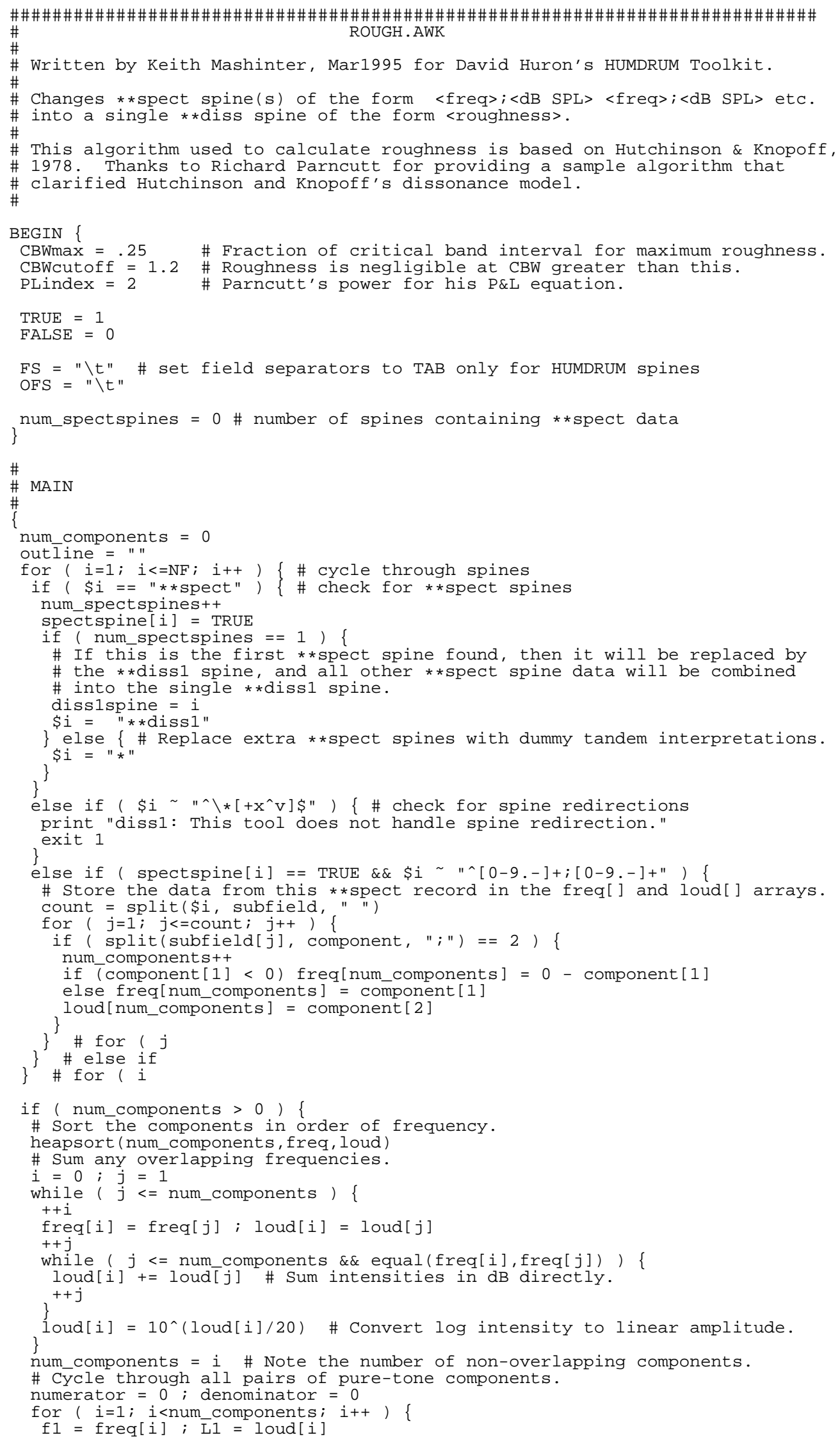




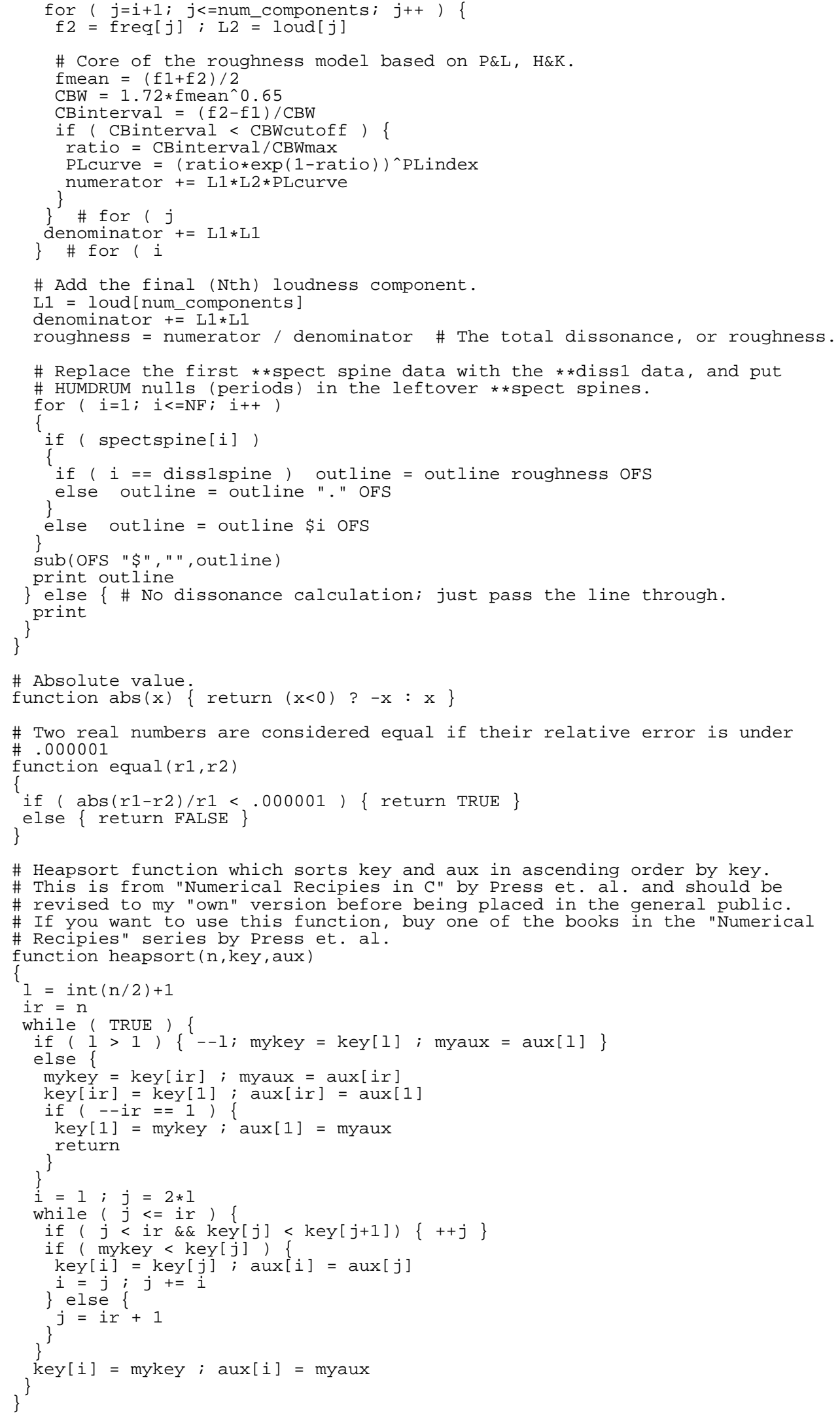

